\title{
A Selective Inhibitor of Ubiquitin-Specific Protease 4 Suppresses Colorectal Cancer Progression by Regulating $\beta$-Catenin Signaling
}

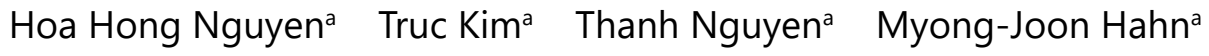 \\ Sun-II Yun ${ }^{a}$ Kyeong Kyu Kimª,c \\ aDepartment of Molecular Cell Biology, Sungkyunkwan University School of Medicine, Suwon, Republic \\ of Korea, 'Institute for Antimicrobial Resistance Research and Therapeutics, Sungkyunkwan University \\ School of Medicine, Suwon, Republic of Korea, 'Samsung Biomedical Research Institute, Samsung \\ Advanced Institute for Health Sciences and Technology, Samsung Medical Center, Sungkyunkwan \\ University School of Medicine, Seoul, Republic of Korea
}

\section{Key Words}

Deubiquitinating Enzyme $\cdot$ USP4 $\bullet$ Noncompetitive Inhibitor $\bullet$ Colorectal Cancer $•$ Beta-catenin

\begin{abstract}
Background/Aims: Dysregulation of deubiquitinating enzymes (DUBs), which regulate the stability of key proteins, has been implicated in many human diseases, including cancers. Thus, DUBs can be considered as potential therapeutic targets for many diseases. Among them, USP4 has been proposed as a promising target for colon cancer drugs since USP4 controls the stability of $\beta$-catenin, a key factor in the Wnt signaling involved in the tumorigenesis of colorectal cancer. However, developing potential DUB inhibitors has been hindered because many DUBs harbor similar active site structures and show broad substrate specificities. Methods: By performing in vitro deubiquitinating activity assays using a chemical library, we identified several potential DUB inhibitors. Among them, only neutral red (NR) showed selective inhibitory activity on USP4 in a cell-based assay system. In colon cancer cells, NR affected the protein stability of $\beta$-catenin, as shown by immunoblotting, and it affected the target gene expression of $\beta$-catenin, as shown by quantitative real-time PCR. NR's potential as an anticancer drug was further estimated by colony formation and cell migration assays and by using a mouse xenograft model. Results: We identified NR as an uncompetitive inhibitor of USP4 and validated its effects in colorectal cancer. NR-treated cells showed decreased $\beta$-catenin stability and reduced expression of $\beta$-catenin target genes. Additionally, treating colon cancer cells with NR significantly reduced colony formation and cell migration, and
\end{abstract}




\section{Cellular Physiology Cell Physiol Biochem 2019;53:157-171 \\ \begin{tabular}{ll|l} 
and Biochemistry $\begin{array}{l}\text { DOl: 10.33594/000000127 } \\
\text { Published online: } 29 \text { June } 2019\end{array}$ & $\begin{array}{l}\text { O } 2019 \text { The Author(s). Published by } \\
\text { Cell Physiol Biochem Press GmbH\&Co. KG }\end{array}$ \\
\cline { 2 - 3 } &
\end{tabular} \\ Nguyen et al.: A Selective Inhibitor of USP4 for the Prevention of Colorectal Cancer}

injecting NR into a mouse xenograft model reduced the tumor volume. Conclusion: The current results suggest that NR could be developed as an anticancer drug targeting USP4, and they support the possibility of developing specific DUB inhibitors as therapeutic agents.

(C) 2019 The Author(s). Published by Cell Physiol Biochem Press GmbH\&Co. KG

\section{Introduction}

Colon cancer, one of most common cancers and the second leading cause of cancer-related deaths in the world, is generally treated with chemotherapeutic drugs. Fluorouracil (5-FU), a genotoxic agent widely used for breast, skin, stomach, gullet, and pancreatic cancer [1], is a representative first-line chemotherapy drug for colon cancer. However, the development of resistance, side-effects, and low efficacy of 5-FU make it necessary to develop more effective and selective chemotherapeutics for colon cancer treatment. Therefore, new targets that are highly expressed and play key roles in colon cancer have been intensively studied.

USP 4 has been proposed as a promising target for colon cancer drugs. USP4 is a member of the ubiquitin-specific protease (USP) family, which is part of the largest subclass of deubiquitinating enzymes (DUBs) [2]. Like other USPs, USP4 uses its deubiquitinating activity to regulate the stability and activation of various proteins within signaling pathways [3]. However, catalytically independent functions of USP4 in the signaling pathway of myoblast differentiation has been also known [4]. It has been validated that USP4 controls the stability of $\beta$-catenin, a key factor in the Wnt signaling involved in the tumorigenesis of colorectal cancer [5]. High expression of USP4 and its substrates $\beta$-catenin and PRL-3 is consistently reported in colon cancer tissue [6,7]. In our previous study, the inhibition of USP4 expression significantly suppressed colon cancer cell activity by regulating the stability and activity of $\beta$-catenin [6]. Additionally, USP4 regulates ARF-BP1, a ubiquitin ligase that decreases the protein stability of p53, which leads to increasing apoptosis, senescence, and oncogenic transformation in colorectal cancer cells [8]. USP4 activity also correlates with other types of cancer cells; for example, USP4 controls the metastatic potential of brain metastatic lung adenocarcinoma through the stabilization of $\beta$-catenin [9]. USP4 deubiquitinates and interacts with TRAF2/TRAF6 during NF- $\mathrm{KB}$ signaling in lung cancer cell migration [10], and it regulates the TAK1 and RIP1 polyubiquitination of TNF $\alpha$-induced NF- $\kappa B$ activation in head and neck squamous cell carcinoma $[11,12]$.

Ubiquitination plays important roles in diverse cellular systems, such as cell growth, DNA damage repair, apoptosis, immune response, and neuron degradation [13]. Ubiquitin has seven internal lysine residues (K6, K11, K27, K29, K33, K48, K63) and the methionine M1 that provide sites for the generation of poly-ubiquitin chains. Among them, K48 and K63 are the two most dominant linkages. The K48-linkage mediates proteasomal degradation, and the K63-chain modulates non-degradative processes such as endocytic trafficking, inflammation, and DNA repair [13]. However, ubiquitination is a reversible process, and the removal of ubiquitin from target proteins is mediated by DUBs. There are about 100 putative DUBs in the human genome, but their functions and substrates are not yet well characterized. Because ubiquitination is important in many key cellular processes either by providing new functions through K63 ubiquitination or by controlling protein stability through K48 ubiquitination, ubiquitination and ubiquitin-processing enzymes are implicated in many human diseases, including cancers. Accordingly, targeting DUBs is emerging as a new therapeutic strategy [14]. For example, auranofin, which shows inhibitory activity on UCHL5 and USP14, is known to selectively suppress tumor growth and induce cancer cell cytotoxicity in acute myeloid leukemia patients [15].

DUBs are classified into five subfamilies based on their sequences and catalytic activities. Among them, the USP subfamily, which contains the largest number of DUBs, commonly has a USP domain, a catalytic domain with Cys (cysteine, $\mathrm{C}$ ) and His (histidine, $\mathrm{H}$ ) in the active site. Accordingly, it is expected that USP inhibitors have rather nonspecific inhibitory activity on various DUBs belonging to the USP subfamily. Thus, although DUB targeting is a promising new drug discovery strategy, the difficulty of developing a selective inhibitor might be a 


\section{Cellular Physiology Cell Physiol Biochem 2019;53:157-171

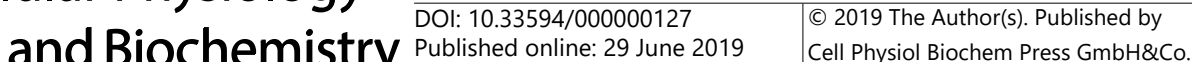 \\ Nguyen et al.: A Selective Inhibitor of USP4 for the Prevention of Colorectal Cancer}

major drawback. Therefore, it is necessary to develop an inhibitor that shows selectivity to a target DUB to enable the development of therapeutics without serious side effects. In this study, we used intensive chemical screening and identified potential DUB inhibitors. Among them, neutral red (NR) showed strong, selective inhibitory activity on USP4 and no inhibitory activity on the other tested DUBs. Using extensive in vitro cellular and in vivo animal model experiments, we validated that NR has the potential to be developed into an anticancer agent targeted specifically to USP4.

\section{Materials and Methods}

\section{Reagents}

All chemicals used in this study were purchased from Sigma-Aldrich (USA), unless otherwise specified. Antibodies against $\beta$-catenin, $\beta$-actin, ubiquitin, and USP4 were purchased from Santa Cruz Biotechnology (USA). Ubiquitin-7-amido-4-methylcoumarin (Ub-AMC) and K48- and K63-linked polyubiquitin chains were purchased from Boston Biochem (USA). A small-molecule library containing 200 chemical compounds was obtained from ChemBridge (USA). Oligodeoxynucleotide primers were synthesized by Macrogen (Korea) and are listed in Supplementary Table S1 (for all supplemental material see www.cellphysiolbiochem.com).

\section{Cell culture and transfection}

HCT116 and 293T cells were cultured in Dulbecco's minimum Eagle's medium (DMEM) supplemented with $10 \%$ fetal bovine serum (FBS). Cells were maintained at $37^{\circ} \mathrm{C}$ in a humidified atmosphere with $5 \% \mathrm{CO}_{2}$. Plasmid transfection into cells was done using TurboFect reagent (Thermo Fisher Scientific, USA) according to the manufacturer's protocol.

\section{Mammalian plasmids}

Plasmids expressing SRT-USP4 were constructed as described previously [6]. Plasmids expressing full-length $\beta$-catenin and the TOP flash reporter system were kindly provided by Dr. Cheol Yong Choi (Sungkyunkwan University, Korea).

\section{Escherichia coli plasmids}

To construct plasmid pVFT1S-USP4 expressing His6-USP4 (residues 8-925), the human USP4 gene was amplified by PCR from the plasmid pET-NKI His-3C-LIC-USP4 [16] using the primer pair 8839F/8840R (Supplementary Table S1). The PCR product was then inserted into the BamHI/XhoI sites of plasmid pVFT1S (Korean Patent No. 1020050051893). Plasmid pVFT1S-YOD1 expressing His6-YOD1 (residues 1-348) was constructed by a similar procedure using the human YOD1 cDNA as a template and the primer pair 2330F/2331R (Supplementary Table S1). The construction of plasmids pVFT1S-USP5 and pVFT1S-UCHL1 was described previously [17]. Plasmid pGEX-2T-USP14 [18] was kindly provided by Dr. Min Jae Lee (Kyung Hee University, Korea). Plasmids expressing glutathione $S$-transferase-ubiquitin-hemagglutinin (GST-UbHA) were kindly provided by Dr. Jin-Hyun Ahn (Sungkyunkwan University, Korea).

\section{Protein expression and purification}

Recombinant USP4, USP5, UCHL1, and YOD1 proteins were expressed and purified using immobilized metal-affinity and size-exclusion chromatography, as described previously [17] with the following minor modifications. Briefly, E. coli Rosetta (DE3) pLysS (Novagen, USA) was used as a host strain for the expression of these proteins. After being purified via Ni-NTA columns, the target proteins were concentrated and loaded onto size-exclusion chromatography columns. The expression and purification of the recombinant USP14 protein followed a similar procedure, previously described by Lee et al. [18]. The GST-Ubi-HA protein was expressed and purified as described previously [17]. All the purified proteins were concentrated, aliquoted, and stored at $-80{ }^{\circ} \mathrm{C}$ until use. The protein purities were more than $90 \%$ as verified by SDS-PAGE analysis. Protein concentrations were determined using the Bradford assay with bovine serum albumin as the standard. 


\section{Cellular Physiology Cell Physiol Biochem 2019;53:157-171 \\ \begin{tabular}{l|l} 
DOI: 10.33594/000000127 & ( 2019 The Author(s). Published by
\end{tabular} \\ and BiOChemistry Published online: 29 June $2019 \quad$ Cell Physiol Biochem Press GmbH\&Co. KG \\ Nguyen et al:: A Selective Inhibitor of USP4 for the Prevention of Colorectal Cancer}

\section{Deubiquitination assays}

All the deubiquitinating reactions were conducted at $25^{\circ} \mathrm{C}$ in $200 \mu \mathrm{L}$ of PBS (pH 7.4) containing $2 \mathrm{mM}$ Dithiothreitol, unless otherwise specified. For the Ub-AMC deubiquitination assays, purified recombinant deubiquitinating protein was pre-incubated with $100 \mu \mathrm{M}$ of small-molecule compound for $10 \mathrm{~min}$. The reaction was initiated by adding Ub-AMC to a final concentration of $0.1 \mu \mathrm{M}$. After a 20-min incubation, the fluorescence of liberated AMC was measured on a Synergy ${ }^{\mathrm{TM}}$ NEO HTS Multi-Mode Microplate Reader (BioTek Instruments, Inc., USA) using the excitation and emission wavelengths of $336 \mathrm{~nm}$ and $441 \mathrm{~nm}$, respectively. For the GST-Ub-HA deubiquitination assay, $200 \mathrm{ng}$ of purified USP4 was pre-incubated with the candidate inhibitor for $20 \mathrm{~min}$. The reaction was initiated by adding $5 \mu \mathrm{g}$ of purified GST-Ub-HA. After a 3-h incubation at $37{ }^{\circ} \mathrm{C}$, the reactions were subjected to SDS-PAGE analysis, and the protein bands were visualized by Coomassie Brilliant Blue staining. The reactivity and specificity of USP4 toward different polyubiquitin chains were examined by incubating $10 \mathrm{nM}$ USP4 with $1.5 \mu \mathrm{g}$ of either K48- or K63-linked polyubiquitin chains (Ub3-7) at $37^{\circ} \mathrm{C}$ for $4 \mathrm{~h}$. To assess the inhibitory effect of NR on USP4, NR was incubated with USP4 prior to the addition of the polyubiquitin chain substrates. The degradation products were analyzed by Coomassie Brilliant Blue-stained SDS-PAGE.

\section{Immunoprecipitation (IP) and immunoblotting}

HCT116 cells were seeded in 60-mm dishes and grown for $16 \mathrm{~h}$, followed by an incubation with various concentrations of NR for an additional $24 \mathrm{~h}$. After being treated with $10 \mu \mathrm{M}$ MG132 for $4 \mathrm{~h}$ to inhibit protein degradation, the cells were collected and lysed in a lysis buffer (50 mM Tris-HCl pH 7.4, 0.1\% Triton X-100, $50 \mathrm{mM} \mathrm{NaF}, 5 \mathrm{mM} \mathrm{Na}_{4} \mathrm{P}_{2} \mathrm{O}_{7}$, and protease inhibitor cocktail). After centrifugation at 12, $000 \mathrm{rpm}$ for $10 \mathrm{~min}$ at $4{ }^{\circ} \mathrm{C}, 500 \mu \mathrm{g}$ of the cell lysate supernatant was incubated with $1 \mu \mathrm{g}$ of the indicated antibody followed by an incubation with Protein A-Sepharose ${ }^{\circledR}$ CL-4B (GE Healthcare, Sweden) for IP. After 6 h, the beads were collected and washed four times in the lysis buffer. Co-immunoprecipitated proteins were separated on 7.5\% SDS-PAGE and detected by Western blot analysis using the indicated antibodies.

\section{$\beta$-catenin stability assay}

HCT116 cells were treated with $100 \mu \mathrm{g} / \mathrm{mL}$ of cycloheximide and harvested at the indicated times to stop protein synthesis. To assess the effect of NR on $\beta$-catenin stability, the cells were treated with $40 \mu \mathrm{M}$ NR in combination with cycloheximide. The levels of $\beta$-catenin protein were determined by western blotting and quantified by densitometric analysis.

\section{Luciferase reporter assay}

The luciferase reporter assay was done as described previously [6] with the following minor modifications. Briefly, the 293T cells were co-transfected with plasmids expressing SRT-USP4, $\beta$-catenin, and the T cell factor reporter system (TOP flash plasmid) for $24 \mathrm{~h}$. The cells were then treated with various concentrations of NR for an additional $24 \mathrm{~h}$ and harvested. Luciferase activity was measured using a dual specific luciferase reporter assay kit (Promega, USA) according to the manufacturer's protocol.

\section{Quantitative real-time PCR}

The qRT-PCR analysis was done as described previously [6] with minor modifications. Briefly, the total cellular RNA was isolated using an RNeasy Mini Kit (Qiagen, Germany) and reverse transcribed using an RNA to cDNA EcoDry Premix (Random Hexamers; Takara, Japan). The expression of the indicated genes was examined using iTaq universal SYBR ${ }^{\circledR}$ Green supermix (Bio-Rad, USA) on a CFX Connect Real-Time PCR Detection System (Bio-Rad, USA). The relative abundance of transcripts was then calculated by the $\Delta \Delta \mathrm{C}_{\mathrm{t}}$ method using CFX Manager software (Bio-Rad, USA). The sequences of primers used for qRT-PCR are shown in Supplementary Table S1.

\section{Cell proliferation assay and cell counting}

To examine the effect of NR on cell proliferation, HCT116 cells were seeded in a 96-well plate at a density of $5 \times 10^{3}$ cells per well in a volume of $100 \mu \mathrm{L}$ followed by treatment with various concentrations of NR for $24 \mathrm{~h}$. The proliferation of HCT116 cells was then examined using an EZ-Cytox Cell Viability kit (Daeil Lab, Korea) according to the manufacturer's instructions. To quantify the proliferating cells, HCT116 cells were seeded in a 24 -well plate at a concentration of $1 \times 10^{4}$ cells $/ \mathrm{mL}$ and grown for $24 \mathrm{~h}$ followed by 


\section{Cellular Physiology Cell Physiol Biochem 2019;53:157-171 \\ \begin{tabular}{l|l}
\hline DOI: 10.33594/000000127 & (c)19 The Author(s). Published by
\end{tabular} \\ and Biochemistry Published online: 29 June $2019 \quad$ Cell Physiol Biochem Press GmbH\&Co. KG \\ Nguyen et al:: A Selective Inhibitor of USP4 for the Prevention of Colorectal Cancer}

treatment with $20 \mu \mathrm{M}$ NR. In addition, to see the combination effect of NR with known cancer drug, NR (10 $\mu \mathrm{M})$ was combined with 5-FU ( $1 \mu \mathrm{M}$ and $3 \mu \mathrm{M}$, Sigma, USA) for the treatment to HCT116 cells. The cells were continuously cultured for 5 days with media changes every $48 \mathrm{~h}$. The viable cells were counted every day using a hemocytometer after being stained with $0.4 \%$ trypan blue solution.

\section{Colony formation assay}

HCT116 cells were seeded into a 6-well plate (500 cells/well) and grown for 2 days followed by treatment with various concentrations of NR for $24 \mathrm{~h}$. The cells were continuously cultured for 14 days with media changes every 2 days. After aspirating the medium, the colonies were stained with a crystal violet solution $(0.05 \% \mathrm{w} / \mathrm{v}$ crystal violet, $1 \%$ formaldehyde, and $1 \%$ methanol in PBS). After a 20 -min incubation at room temperature, the plate was rinsed with distilled water and allowed to air dry overnight. The plate was then photographed, and the number of colonies was counted using ImageJ software [19].

Wound-healing assay

HCT116 cells were seeded in a 12-well plate and maintained until confluent. The confluent cell monolayer was scratched in a line with a sterilized $20 \mu \mathrm{L}$ plastic pipette tip to form a wound area that we then washed twice with serum-free medium to remove the detached cells. The cells were incubated with serum- and NR-containing medium for $24 \mathrm{~h}$. The plate was photographed, and the closure of the wound area was evaluated using ImageJ software [19].

\section{Cell migration assay}

The migration assay was performed in a 24-well Matrigel migration chamber plate (Millipore, USA) according to the manufacturer's protocol. Briefly, after adding $0.3 \mathrm{~mL}$ of culture medium containing $1 \times 10^{5}$ HCT116 cells to the upper chambers, $0.5 \mathrm{~mL}$ of serum-containing medium supplemented with various concentrations of NR was placed in the bottom chamber. After $24 \mathrm{~h}$, the migrated cells were stained and photographed under a microscope. To quantify the amount of migration, the stained cells were extracted, and the optical density at $560 \mathrm{~nm}$ was measured using a plate reader.

\section{In vivo xenograft model}

To evaluate the inhibitory effect of NR on in vivo tumor growth, $0.1 \mathrm{~mL}$ of HCT116 cells $\left(3 \times 10^{6}\right.$ cells $)$ were subcutaneously injected into 6-week-old BALB/c nude mice (SPF grade) using 27G syringe needles. After 5 days, the mice were divided into four treatment groups, and each group $(n=8)$ was intraperitoneally injected with either PBS (as a control), NR (10 mg/kg or $50 \mathrm{mg} / \mathrm{kg}$ ), or 5 -FU (25 mg/kg) every 3-4 days for 5 weeks. The body weight and tumor size were monitored every day. When the tumor size of the control group (PBS treatment) was approximately $1,000 \mathrm{~mm}^{3}$, the mice were sacrificed, and the tumors were collected for assessment. All manipulations involving live mice were approved by the Institutional Animal Care and Use Committee (IACUC) of Seoul National University (Korea).

\section{Immunohistochemistry (IHC) staining}

For IHC testing, the xenograft tumor specimens were stained with either anti-rabbit polyclonal $\beta$-catenin or anti-mouse USP4 (Santacruz, USA). After deparaffinization and rehydration, formalin-fixed, paraffin-embedded xenograft tumor sections were incubated in PBS containing $0.3 \% \mathrm{H}_{2} \mathrm{O}_{2}$ for 10 min at room temperature followed by an antigen retrieval step carried out by boiling the slides in $0.01 \mathrm{M}$ citrate buffer for $5 \mathrm{~min}$. The sections were then washed in $50 \mathrm{mM}$ Tris- $\mathrm{HCl} \mathrm{pH} 7.6$ and $0.05 \%$ Tween20 for $2 \mathrm{~min}$. To block nonspecific binding, all the sections were treated with PBS containing 2\% BSA and 2\% goat serum for $30 \mathrm{~min}$ at room temperature. The slides were then incubated with a primary antibody (rabbit polyclonal anti- $\beta$-catenin antibody or mouse anti-USP4 antibody) in the blocking solution at $4{ }^{\circ} \mathrm{C}$ overnight. The reaction was stopped by rinsing the section with PBS. The slides were then incubated with a Dako EnVision+ System HRP-labeled polymer anti-rabbit or anti-mouse (Agilent, USA) and treated with diaminobenzidine substrate to visualize the positive cells. Finally, the sections were counterstained with hematoxylin before being mounted for light microscopy examination. 


\section{Cellular Physiology Cell Physiol Biochem 2019;53:157-171

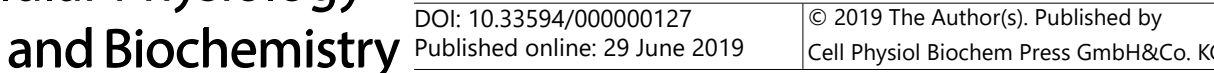 \\ Nguyen et al: A Selective Inhibitor of USP4 for the Prevention of Colorectal Cancer}

Statistical analysis

All experiments were conducted at least in triplicate. The results are expressed as mean \pm standard deviation (SD). Statistically significant differences were analyzed by the Student's $t$-test in one variable and by analysis of variance (ANOVA) test in over two variables between two groups; ${ }^{*} \mathrm{p}<0.05,{ }^{* *} \mathrm{p}<0.01$, ${ }^{* * *} \mathrm{p}<0.001$, and ${ }^{* * * *} \mathrm{p}<0.0001$ were all considered to be statistically significant.

\section{Results}

Neutral red was identified as an uncompetitive inhibitor of USP4

For the enzyme activity assay, recombinant USP4 was overexpressed in E.coli and prepared with high purity. Ubiquitin (Ub)-AMC was used as an artificial substrate for the DUB assay. The fluorescence intensity of the AMC hydrolyzed from Ub-AMC was interpreted as the DUB activity of USP4. In our chemical screening using a small-molecule library, we identified several potent inhibitors of USP4 (Supplementary Fig. S1A and S1B, Supplementary Table S2). To discover a USP4-specific inhibitor, we measured their inhibitory activity against USP4, YOD1, USP5, UCHL1, and USP14. In that way, we identified NR (3-amino-7dimethylamino-2-methyl-phenazine hydrochloride, Fig. 1A) and 2, 3-diaminophenazine (Supplementary Fig. S3A) as candidates because they showed the highest inhibitory activity on USP4 but no effective activity on the other DUBs (Fig. 1B, Supplementary Fig. S3D). However, 2, 3-diaminophenazine showed no inhibitory activity in a further cellular assay (Supplementary Fig. S3G and S3H), so we selected NR as a potential inhibitor candidate for further investigation.

The IC50 and IC90 values for NR against USP4 were $50 \mu \mathrm{M}$ and $200 \mu \mathrm{M}$, respectively, when Ub-AMC was used as the substrate (Fig. 1C). Next, we confirmed the DUB activity of USP 4 by examining the release of HA from GST-Ub-HA using SDS-PAGE. When $200 \mu \mathrm{M}$ NR was added, HA-release was completely inhibited (Fig. 1D). We examined the inhibitory activity of NR on USP4 by monitoring the monomerization of K48 and K63 poly-Ub chains because USP4 was reported to have deubiquitinase activity on both the K48 and K63 linkages [12, 20]. When NR was added to the mixture containing USP4 together with poly-K48-ubiquitins or poly-K63-ubiquitins, the amount of $8 \mathrm{kDa}$ ubiquitin monomer decreased in a concentrationdependent manner (Fig. 1E). These results revealed that NR inhibits USP 4 activity on various substrates.

To examine the inhibitory mechanism of NR against USP4, $V_{0}$ value was investigated with various amounts of Ub-AMC $(1-250 \mu \mathrm{M})$ and NR $(0-15 \mu \mathrm{M})$ by fixing the concentration of USP4 at $400 p \mathrm{M}$ (Fig. 1F). That analysis confirmed that NR affects both $\mathrm{K}_{\mathrm{m}}$ and $\mathrm{V}_{\text {max' }}$ decreasing $\mathrm{K}_{\mathrm{m}}$ from 112.2 to 19.2 and $\mathrm{V}_{\max }$ from 0.139 to 0.0196 (Fig. $1 \mathrm{~F}$ ). That result indicates that NR preferentially inhibits USP4 activity by binding to the USP4 complex with Ub as an uncompetitive inhibitor of USP4.

NR inhibits the deubiquitinating activity of USP4 in colon cancer cells

After confirming that NR works as an uncompetitive inhibitor of USP4 in vitro, we investigated whether its inhibitory activity occurred in HCT116 colon cancer cells by examining the cellular deubiquitinating activity of USP4 upon NR treatment. HCT116 cells were co-transfected with HA-Ub and SRT-USP4, followed by treatment with NR for $24 \mathrm{~h}$. Then we used IP to examine the amounts of ubiquitinated proteins. This experiment confirmed that USP4 reduced the number of K48- and K63-linked polyubiquitin chains, which was reversed by the NR treatment (Fig. 2A).

After confirming the inhibitory activity of NR on USP4 in HCT116 cells, we investigated the effects of NR on the cellular substrates of USP4. Previously, it was shown that $\beta$-catenin is a substrate of USP4, and the enhanced stability it gains when USP4 is highly expressed contributes to the progress of colon cancers [6]. Therefore, we investigated whether NR affects the stability of $\beta$-catenin by inhibiting USP 4 activity in HCT116 cells. As expected, NR treatment dose-dependently decreased the protein level of $\beta$-catenin (Fig. 2B). Next, to 


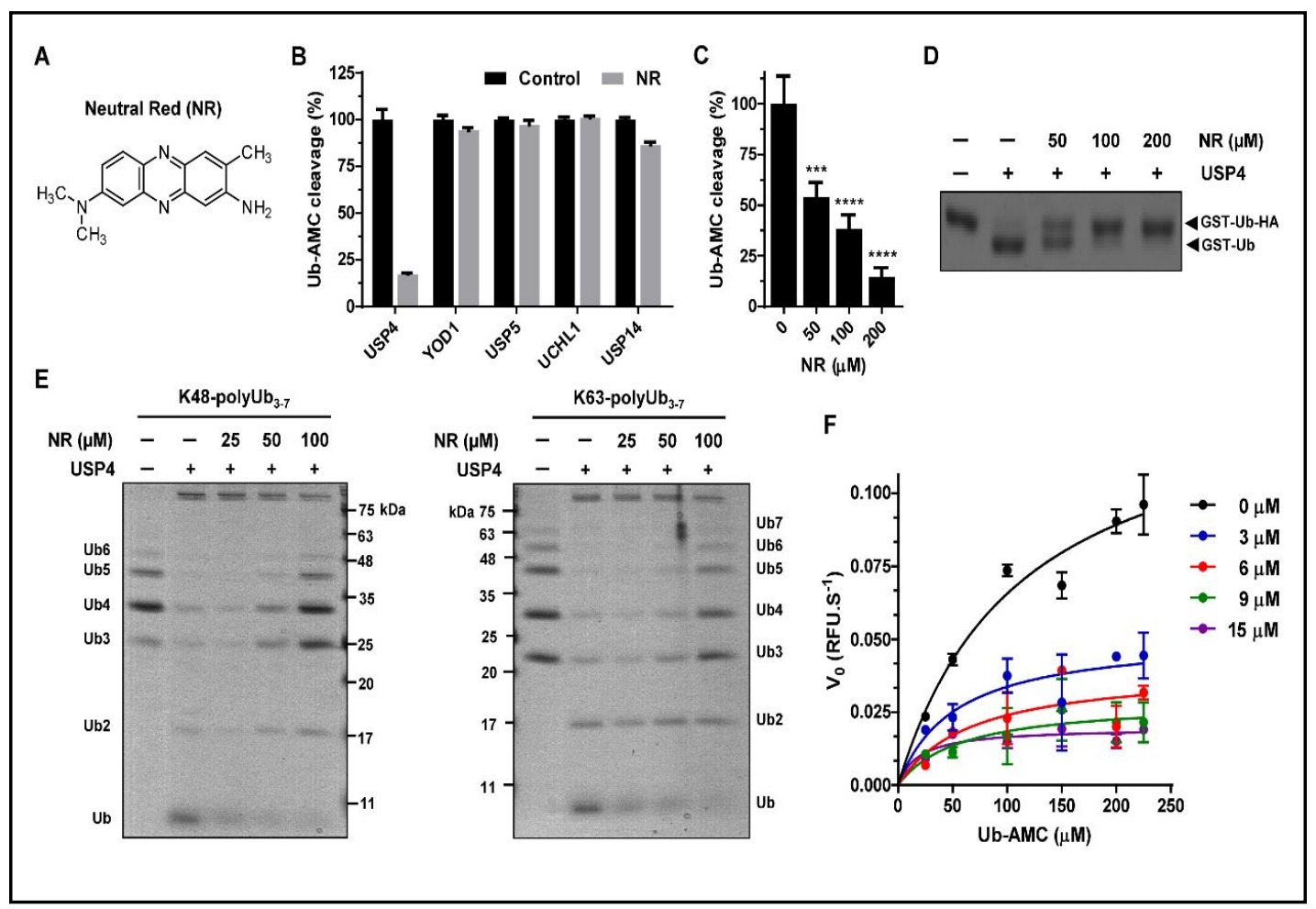

Fig. 1. NR is a small-molecule inhibitor of USP4 activity. (A) Chemical structure of NR. (B) The DUB activity of USP4, YOD1, USP5, UCHL1, and USP14 with and without $200 \mu \mathrm{M}$ of NR was compared using Ub-AMC deubiquitination assays in which AMC hydrolyzed from the Ub-AMC substrate was measured as fluorescence at an excitation wavelength of $336 \mathrm{~nm}$ and an emission wavelength of $441 \mathrm{~nm}$. (C) The effect of various concentrations of NR on the deubiquitinating activity of USP4 was measured using UbAMC deubiquitination assays. Error bars represent $S D,{ }^{* * *} p<0.001,{ }^{* * * *} p<0.0001$. (D) The effect of various concentrations of NR on the deubiquitinating activity of USP4 was measured by monitoring the band shift from GST-Ub-HA to GST-Ub. (E) The effect of various concentrations of NR on the deubiquitinating activity of USP4 was measured by monitoring Ub cleavage from K48- (left) and K63- (right) linked polyubiquitin chains. The activity was examined on 15\% SDS-PAGE gel with Coomassie Blue staining. (F) MichaelisMenten plot for calculating the DUB activity of USP4. Hydrolysis Ub-AMC assays were performed after USP4 reacted with Ub-AMC $(0-225 \mathrm{nM})$ in the presence of $\mathrm{NR}$ at various concentrations $(0-15 \mu \mathrm{M})$.

investigate whether NR affects the protein stability of $\beta$-catenin by inhibiting USP4, we first exposed cells to cycloheximide, a protein synthesis inhibitor, with and without NR treatment (Fig. 2C). As expected, the $\beta$-catenin level was much reduced in the presence of NR compared to the control (cycloheximide treatment without NR, Fig. 2C, top). Then the same experiments were performed after USP4 overexpression (Fig. 2C, bottom). Consistently, NR reduced the $\beta$-catenin level that was enhanced by USP4 expression. These results clearly explain that USP 4 stabilizes $\beta$-catenin, but inhibition of USP 4 by NR reduces the stability of $\beta$-catenin in colon cancer cells. Overall, it can be concluded that $\beta$-catenin stability can be regulated by NR through USP4 inhibition.

Because USP4 works as a deubiquitinase of $\beta$-catenin via direct interaction [6], we further checked the effects of NR by examining the ubiquitination level of $\beta$-catenin. HCT116 cells were or were not treated with NR for $24 \mathrm{~h}$ and then treated with MG132 to inhibit the proteasomal degradation of ubiquitinated $\beta$-catenin. As shown in Fig. $2 \mathrm{D}$, the amount of ubiquitinated $\beta$-catenin was enhanced by NR treatment, suggesting that NR interrupts the deubiquitinating activity of USP4 on $\beta$-catenin. To further investigate whether NR treatment can affect the interaction between USP4 and its substrates in cells, we used IP to evaluate the binding of $\beta$-catenin to UPS4. When HCT116 cells were exposed to NR at 


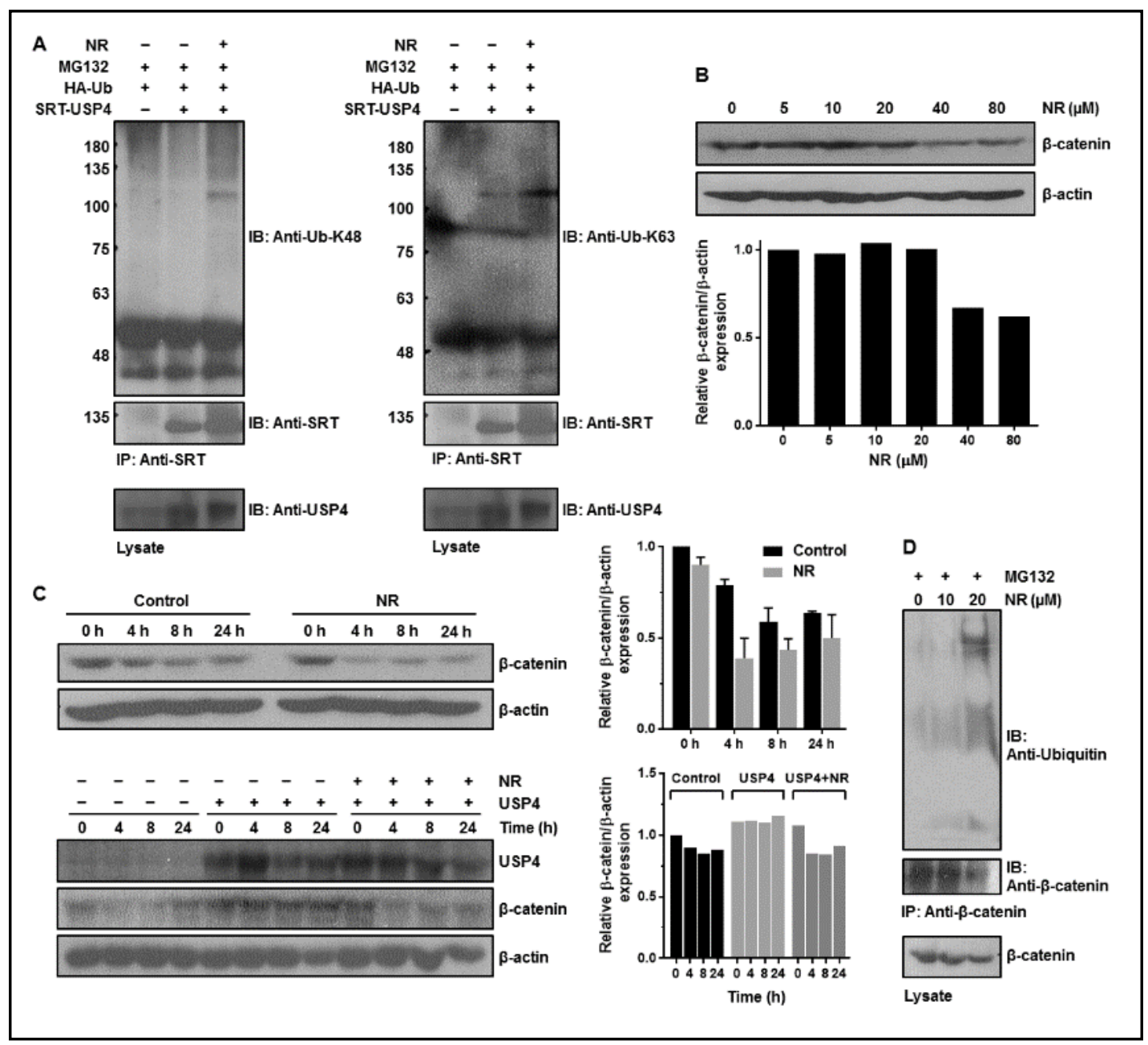

Fig. 2. NR inhibits the deubiquitinating activity of USP4 in HCT116 cells. (A) HCT116 cells were transfected with SRT-USP4 and HA-ubiquitin (HA-Ub). Transfected cells were or were not treated with $40 \mu \mathrm{M}$ NR for $24 \mathrm{~h}$, and then $10 \mu \mathrm{M}$ MG132 was added for $4 \mathrm{~h}$ to block protein degradation. Ubiquitinated proteins were precipitated by anti-SRT antibody and detected using anti-K48 (left) and anti-K63 (right) ubiquitin antibodies. (B) HCT116 cells were treated with the indicated concentrations of NR for $24 \mathrm{~h}$, and endogenous $\beta$-catenin expression was observed by immunoblotting (top). The immunoblot band of $\beta$-catenin was quantified by densitometry (bottom). The protein level of $\beta$-catenin was normalized to $\beta$-actin. (C) HCT116 cells were treated with $100 \mu \mathrm{g} / \mathrm{ml}$ cycloheximide (CHX) with or without $40 \mu \mathrm{M}$ NR (top). Empty vector (control) and SRT-USP4 plasmid was transiently transfected into HCT116 cell. At $24 \mathrm{~h}$ after transfection, the cells were treated with $100 \mu \mathrm{g} / \mathrm{ml} \mathrm{CHX}$ with or without $40 \mu \mathrm{M}$ NR for indicated times (bottom). The cells were lysed and the amount of $\beta$-catenin was analyzed by immunoblotting (left) and the expression amount was quantified by densitometry (right). (D) HCT116 cells were or were not treated with $10 \mu \mathrm{M}$ or $20 \mu \mathrm{M}$ NR for $24 \mathrm{~h}$. The cells were then treated with $10 \mu \mathrm{M}$ MG132 for $4 \mathrm{~h}$ prior to harvest. Endogenous ubiquitinated $\beta$-catenin was precipitated using anti- $\beta$-catenin antibody and then immunoblotted with ubiquitin antibody.

various concentrations during USP4 overexpression, the amount of $\beta$-catenin binding to USP 4 was enhanced in a concentration-dependent manner (Supplementary Fig. S2). This result indicates that NR did not interrupt the interaction between USP4 and $\beta$-catenin but instead stabilized the enzyme-substrate complex. Under the same condition, we confirmed that the DUB activity of USP4 was clearly inhibited by NR (Fig. 2). These results together suggest that NR is an uncompetitive inhibitor of USP4. 
NR controls $\beta$-catenin activity in colon cancer cells

After we validated that NR affects $\beta$-catenin stability by inhibiting USP4, we investigated the cellular effect of NR on $\beta$-catenin by examining the transcriptional activity of $\beta$-catenin through a TOP flash reporter assay. USP4 and $\beta$-catenin were co-transfected with a reporter plasmid (TOP flash) in 293T cells, followed by treatment with NR at various concentrations. As shown in Fig. 3A, USP4 overexpression enhanced $\beta$ - c a te n in - in d u c e d transcriptional activity, but when it was combined with NR treatment, the transcriptional activity was repressed in a dosedependent manner. Therefore, NR regulates the transcriptional activity of $\beta$-catenin by controlling its stability. To corroborate this interpretation, we evaluated the mRNA expression of Cyclin D1 and Axin2, $\beta$-catenin targets, in HCT116 cells treated with NR. As shown in Fig. 3B, USP4 overexpression significantly induces the expression of Cyclin D1 and Axin2 dose-dependently, but their expression was reduced by NR treatment. Inhibition of Cyclin D1 and Axin2 expression by NR treatmentwasalso observed even in the absence of USP4 overexpression (Fig. 3B). However, the mRNA expression of $\beta$-catenin and

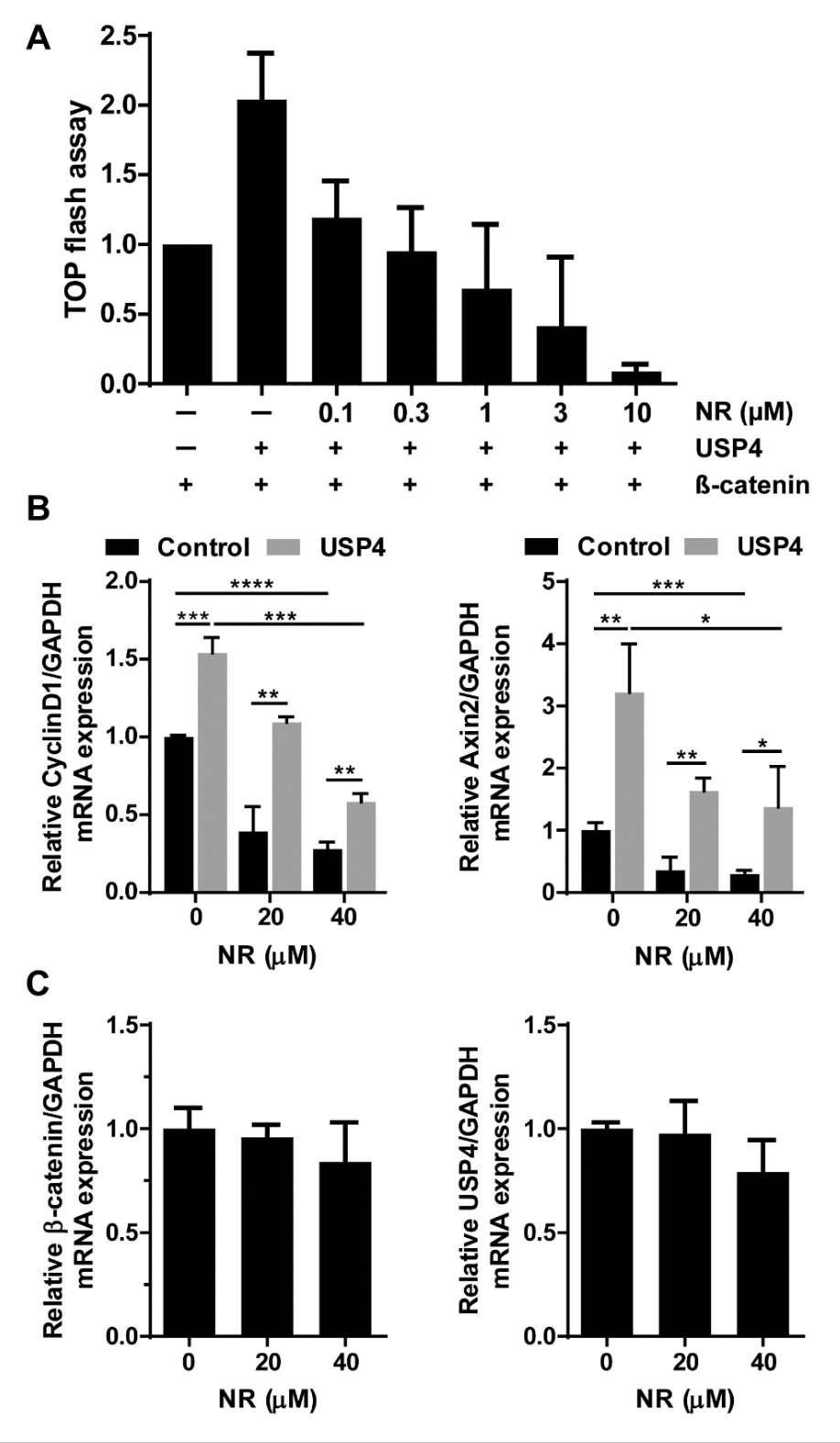

Fig. 3. NR controls $\beta$-catenin activity and the target gene expression of $\beta$-catenin. (A) $293 \mathrm{~T}$ cells were co-transfected with $\beta$-catenin and USP4 to evaluate the effect of NR on $\beta$-catenin-mediated transcriptional (TOP flash) activity, and then NR was added at the indicated concentrations for $24 \mathrm{~h}$. (B, C) HCT116 cells were exposed to NR $(0 \mu \mathrm{M}, 20 \mu \mathrm{M}$, and $40 \mu \mathrm{M}$ ) for $24 \mathrm{~h}$ with (B) or without (C) transfection of empty vector or SRT-USP4 plasmid. The mRNA expression of cyclin D1 and Axin2 (B), and $\beta$-catenin and USP4 (C) was quantified by real-time PCR and normalized to GAPDH. Error bars represent SD, ${ }^{* *} \mathrm{p}<0.01,{ }^{* * *} \mathrm{p}<0.001$, $* * * * \mathrm{p}<0.0001$. USP4 was not significantly affected by NR treatment (Fig. 3C), suggesting that NR controls the protein stability of $\beta$-catenin and the protein activity of USP 4 instead of affecting their gene expression. 


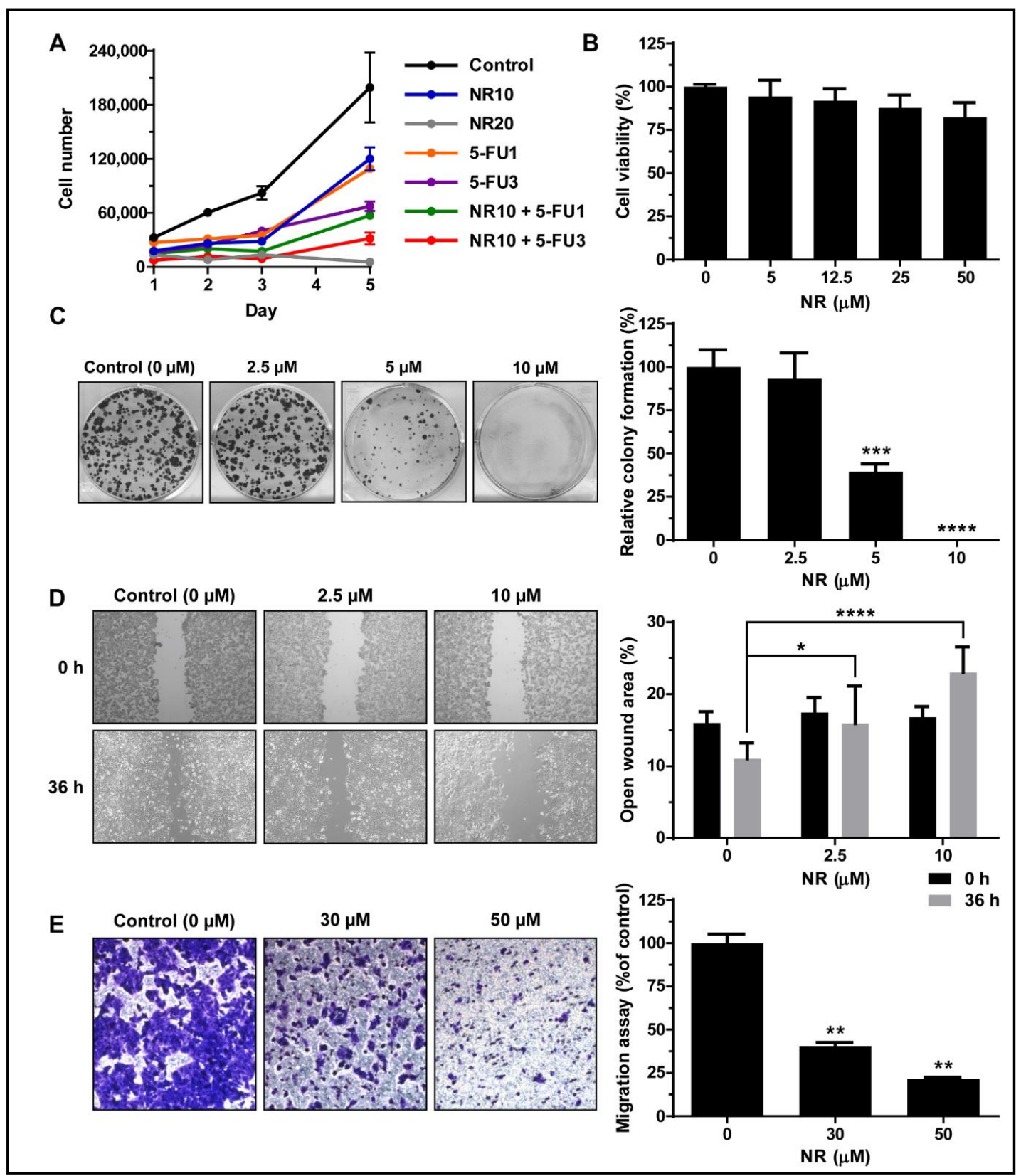

Fig. 4. NR inhibits the proliferation and migration of HCT116 cells. (A) The effect of drug treatment on cell grwoth was analyzed by counting cell numbers. HCT116 cells were grown for 5 days followed by treatment with $10 \mu \mathrm{M}$ and $20 \mu \mathrm{M}$ of $\mathrm{NR}$, or $1 \mu \mathrm{M}$ and $3 \mu \mathrm{M}$ of 5 -FU from 0 to 5 days. For exmaming the combination effect, cells were co-treated with $10 \mu \mathrm{M}$ NR and 5-FU (1 $\mu \mathrm{M}$ and $3 \mu \mathrm{M})$. (B) HCT116 cells were treated with the indicated doses of NR for $24 \mathrm{~h}$, and cell viability was measured by WST assay. (C) HCT116 cells were treated with various concentrations of NR for $24 \mathrm{~h}$, and the cells were cultured for 14 days before staining with crystal violet solution (left). The stained colonies were counted, and the relative amounts are shown in a graph (right). (D) HCT116 cells were treated with different doses of NR for $36 \mathrm{~h}$, and cell migration was examined by a wound-healing assay. Images of the migrated cells were taken under $\times 200$ magnification (left), and the open wound area was measured using ImageJ and presented in a bar graph (right). Error bars represent $\mathrm{SD},{ }^{*} \mathrm{p}<0.05,{ }^{* *} \mathrm{p}<0.01,{ }^{* * *} \mathrm{p}<0.001,{ }^{* * * *} \mathrm{p}<0.0001$. (E) HCT116 cells were treated with $30 \mu \mathrm{M}$ or $50 \mu \mathrm{M}$ NR for $24 \mathrm{~h}$ and then performed transwell migration assays (left), and the relative percentage of migratory cells was calculated (right). Error bars represent SD, ${ }^{*} \mathrm{p}<0.05,{ }^{* *} \mathrm{p}<0.01$, ${ }^{* * *} \mathrm{p}<0.001,{ }^{* * * *} \mathrm{p}<0.0001$. 


\section{Cellular Physiology Cell Physiol Biochem 2019;53:157-171

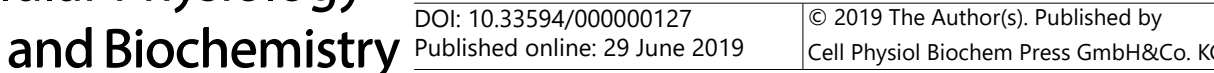 \\ Nguyen et al.: A Selective Inhibitor of USP4 for the Prevention of Colorectal Cancer}

\section{NR inhibits the cell proliferation and migration of colon cancer cells}

$\beta$-catenin plays a key role in the migration and proliferation of colon cancer cells. We thus expected NR to inhibit those activities in cancer cells. To test that hypothesis, we measured cell proliferation activity by counting cell numbers for 5 days after adding NR to HCT116 cells. Compared to the NR effect, we used 5-FU, a chemotherapeutic agent for colon cancer treatment, on cancer cell proliferation (Fig. 4A). Each NR and 5-FU treatment inhibited the cell growth of HCT116 in a dose-dependent manner. Furthermore, the combination treatment of NR and 5-FU clearly decreased the cell numbers at Day 5; NR 60.2\%, NR+1uM 5-FU 28.7\%, and NR+3uM 5-FU 15.88\% compared to control at Day 5 (100\%) (Fig. 4A).

The effect of NR on colon cancer cells was also investigated using a colony formation assay. In line with the former results, colony formation was significantly inhibited by treating HCT116 cells with NR (Fig. 4C). Because NR showed no toxicity in HCT116 cells (Fig. 4B), we conclude that the effect of NR on colon cancer cells is not caused by its toxic effect on cell viability but by its inhibitory activity on USP 4 and $\beta$-catenin.

To investigate whether NR could inhibit the metastatic activity of colon cancer cells, we measured wound healing and migration activity in HCT116 cells. During wound healing, cells treated with $10 \mu \mathrm{M}$ NR showed significantly reduced cell movement $(23 \%$ of open wound area) compared with the control (11\% of open wound area) (Fig. 4D). Consistently, NR treatment also significantly decreased the number of migrating cells (Fig. 4E). Therefore, we conclude that NR inhibits the migration of HCT116 cancer cells.

\section{NR inhibits tumor cell growth in mice}

Having confirmed that NR treatment inhibits the activity of HCT116 cells, we next examined whether NR has antitumor activity in a mouse xenograft model. For comparison, we used 5-FU as a positive control. The xenograft tumors in mice were made using HCT116 colon cancer cells. PBS (control), NR $(25 \mathrm{mg} / \mathrm{kg}, 50 \mathrm{mg} / \mathrm{kg}$ ), or $5-\mathrm{FU}(25 \mathrm{mg} / \mathrm{kg})$ was injected intraperitoneally every 3 days. After 40 days, both the NR and 5-FU treatments had significantly inhibited the growth of the xenograft tumors compared to the control: by $60 \%$ in the NR $50 \mathrm{mg} / \mathrm{kg}$ group and $28 \%$ in the 5 -FU group (Fig. 5A). Moreover, both tumor volume and tumor weight were much less than the control with NR treatment at $50 \mathrm{mg} / \mathrm{kg}$ or $5-\mathrm{FU}$ (Fig. 5B and C), but there were no apparent changes in body weight (Fig. 5D).

The amount of $\beta$-catenin and USP4 in the xenograft tumors was estimated by IHC. Both NR and 5-FU treatment decreased the expression of $\beta$-catenin but not that of USP4 (Fig. 5E). Consistently, Western blotting confirmed that the amount of $\beta$-catenin was clearly reduced in the tissues treated with NR and 5-FU (Fig. 5F). The in vivo xenograft results thus confirm that the effects of NR in the mouse model are well consistent with those in the in vitro cell migration assay. Therefore, we suggest that cellular NR treatment can inhibit tumorigenesis by regulating $\beta$-catenin expression in colon cancer.

\section{Discussion}

The overexpression of USP4 in colon cancer suggests that USP4 acts as tumorpromoting protein in colon cancer $[6,7,21]$. In this study, we identified NR as an inhibitor of USP4 by screening small-molecule compound libraries (Fig. 1). We confirmed that NR works as an uncompetitive inhibitor of USP4, i.e., NR acts selectively on UPS4, not on other USPs. Furthermore, we showed that NR suppresses the tumorigenic progress of colon cancer cells (Fig. 4) by inhibiting the deubiquitinating activity of USP4, which decreases the protein stability and activity of $\beta$-catenin (Fig. 2 and 3). Finally, we demonstrated that NR has potential for use as a cancer drug by showing that intraperitoneal-injection of NR into a colon cancer cell-induced xenograft mouse model reduced tumor growth and weight compared with control mice and decreased the expression of $\beta$-catenin in tumor tissue (Fig. $5)$. 


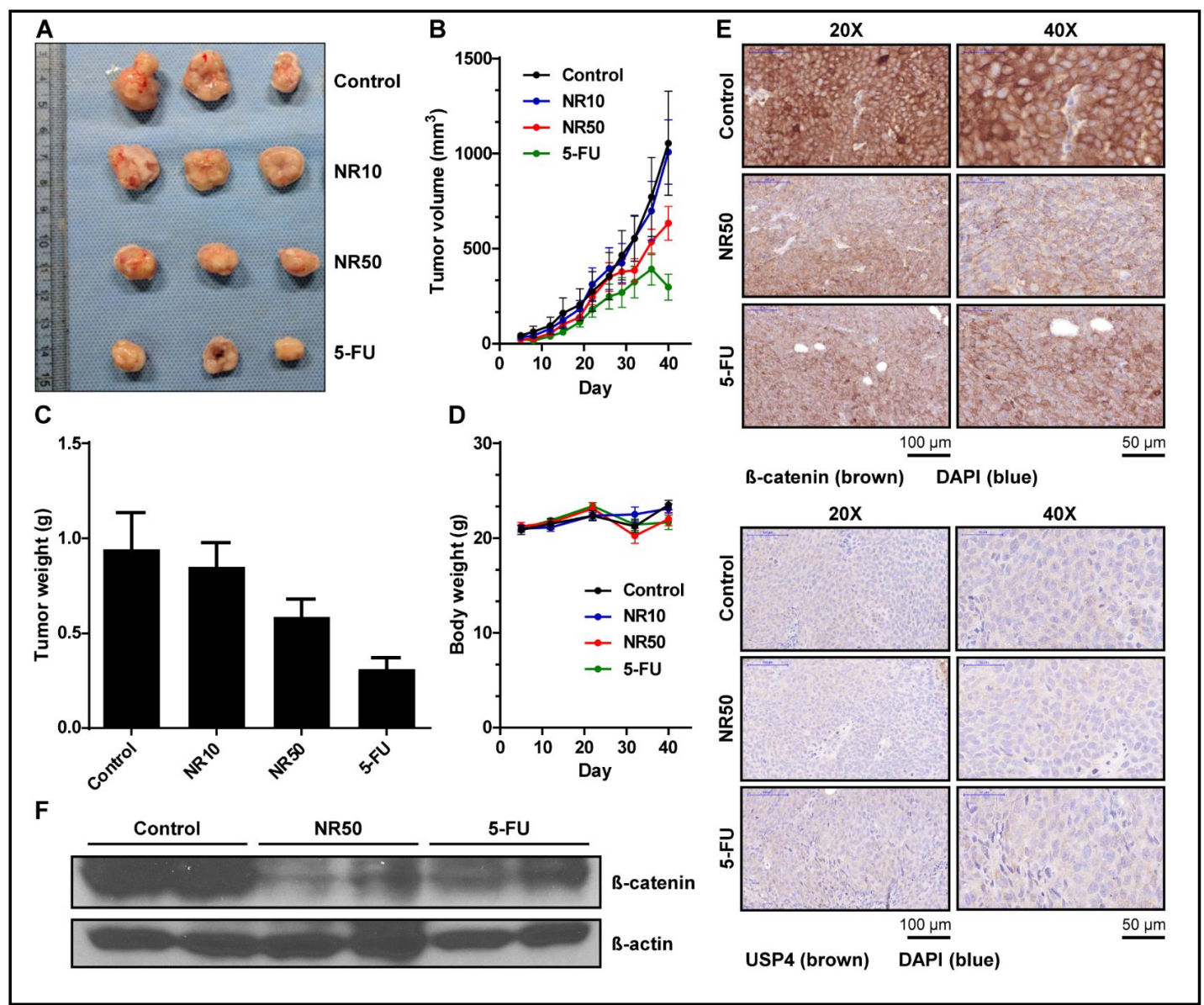

Fig. 5. NR inhibits tumor growth in HCT116 xenografts. (A) Representative picture of tumors excised from mice injected with PBS, $10 \mathrm{mg} / \mathrm{kg}$ NR (NR10), $50 \mathrm{mg} / \mathrm{kg}$ NR (NR50) and $25 \mathrm{mg} / \mathrm{kg}$ 5-FU (5-FU). (B) The volume of tumors was measured during indicated periods. Data are tumor volume mean \pm SD, and each groups were eight mice ( $\mathrm{n}=8$ ). Two of eight animals in 5-FU group were unexpectedly died after 30 days. Survival rate is $100 \%$ (8/8) in control, $100 \%$ (8/8) NR50, and 75\% (6/8) in 5-FU. (C) Average tumor weight excied from each group. (D) Average body weight were recorded for 40 days after drug injection. Error bars represent SD, ${ }^{*} \mathrm{p}<0.05$. (E) Immunohistochemistry of $\beta$-catenin (top) and USP4 (bottom) levels in the xenograft tumor tissues of mice were observed under $\times 20$ and $\times 40$ magnification and added scale bar in the bottom. (F) Expression of $\beta$-catenin protein in the xenograft tumors. Two representative tumor tissues from each group (control, NR50, and 5-FU) were randomly homogenized in lysis buffer and subjected to Western blotting with specific anti- $\beta$-catenin antibody. $\beta$-actin was used as a loading control.

The high expression and dysregulation of $\beta$-catenin, which is related to the Wnt signaling pathway, is involved in various human diseases, especially colon cancers [22]. Even though many inhibitors that interrupt Wnt/ $\beta$-catenin signaling have been identified and studied as candidates for colon cancer treatment [23], no inhibitors that control the protein stability of $\beta$-catenin as a way of blocking Wnt signaling have previously been reported. In this study, we propose a new approach to the treatment of colon cancer: reducing the protein stability of $\beta$-catenin by inhibiting USP 4 activity. We previously studied $\beta$-catenin as a substrate of USP4 $[6,9]$. In this study, we demonstrated that treating colon cancer cells with NR reduced the protein stability and functional activity of $\beta$-catenin by impairing the deubiquitinating activity of USP4 (Fig. 2 and 3) and thereby promoting the ubiquitination and proteasomal degradation of endogenous $\beta$-catenin. Consistently, NR treatment suppressed both the proliferation and migration of colon cancer cells (Fig. 4), activities regulated by the ubiquitination of $\beta$-catenin. 


\section{Cellular Physiology Cell Physiol Biochem 2019;53:157-171 \\ \begin{tabular}{cc|c} 
DOl: 10.33594/000000127 & O 2019 The Author(s). Published by \\
Cell Physiol Biochem Press GmbH\&Co. KG
\end{tabular}

$\mathrm{NR}$, a eurhodin dye containing aminophenazine structure, is generally used to monitor intracellular $\mathrm{pH}$, stain lysosomes in viable cells, and measure cell cytotoxicity in many biomedical and environmental applications [24]. The primary target of NR binding in cells is a riboflavin binding protein [25]. However, its cellular and clinical relevance had not been previously reported. In this study, we demonstrated that NR binds to the USP4-substrate complex as an uncompetitive inhibitor and prevents cancer progress by reducing the stability of $\beta$-catenin. We hypothesize that the aminophenazine structure of NR is crucial to USP4 inhibition because 2, 3-diaminophenazine and NR share both aminophenazine and inhibitory activity on USP4 (Supplementary Fig. S3). NR showed higher cellular activity than 2,3-diaminophenazine, so we investigated NR as a USP4 inhibitor. It is unclear why NR showed better cellular activity than the other aminophenazine compounds with similar in vitro inhibitory activity. NR does enter the cell interior through an endocytic process called micropinocytosis [26] as well as direct diffusion, so a higher cellular uptake rate might be why NR showed the highest cellular activity.

Although we showed NR as a USP4-specific inhibitor by elucidating how NR controls $\beta$-catenin stability, and we demonstrated that NR treatment can effectively suppress cancer progress using a xenograft model, NR cannot be used as a cancer drug in its current form because its IC50 value is relatively high. Nonetheless, this study is meaningful because we have validated a new drug-targeting strategy by demonstrating that it is possible to selectively inhibit a specific DUB by using an uncompetitive inhibitor, even though many DUBs share their active sites. Indeed, the well-characterized DUBs, such as USP7 and USP14, have been proposed as good pharmacological targets for cancer treatment $[27,28]$ so the strategy proposed in this study can be applied to other USPs involved in various human cancers.

\section{Conclusion}

We identified NR an uncompetitive inhibitor of USP4 and NR treatment resulted in suppressing $\beta$-catenin and Wnt signaling in colon cancer cells and decreasing colon tumor growth in in vivo mouse model. Therefore, the current results of this study may confer a plausible strategy with a specific inhibitor targeting USP4 for colon cancer treatment, and they could support the possibility of developing other specific DUB inhibitors as therapeutic anti-cancer drugs.

\section{Acknowledgements}

We thank Dr. Titia Sixma, Dr. Cheol Yong Choi, Dr. Min Jae Lee, and Dr. Jin-Hyun Ahn for their kind provision of the plasmid constructs used in this study. This work was supported by National Research Foundation of Korea (NRF-2016R1A6A3A11930924 and 2019M3A9A8067081).

\section{Disclosure Statement}

The authors declare no conflicts of interest. 


\section{Cellular Physiology Cell Physiol Biochem 2019;53:157-171 \begin{tabular}{l|l} 
DOI: 10.33594/000000127 & (c)19 The Author(s). Published by
\end{tabular} and Biochemistry Published online: 29 June $2019 \quad$ Cell Physiol Biochem Press GmbH\&Co. KG \\ Nguyen et al:: A Selective Inhibitor of USP4 for the Prevention of Colorectal Cancer}

\section{References}

1 Sánchez-Gundín J, Fernández-Carballido AM, Martínez-Valdivieso L, Barreda-Hernández D, Torres-Suárez AI: New Trends in the Therapeutic Approach to Metastatic Colorectal Cancer. Int J Med Sci 2018;15:659665.

2 Clague MJ, Barsukov I, Coulson JM, Liu H, Rigden DJ, Urbé S: Deubiquitylases from genes to organism. Physiol Rev 2013;93:1289-1315.

3 Zhao B, Schlesiger C, Masucci MG, Lindsten K: The ubiquitin specific protease 4 (USP4) is a new player in the Wnt signalling pathway. J Cell Mol Med 2009;13:1886-1895.

4 Yun SI, Kim K: Ubiquitin-specific protease 4 (USP4) suppresses myoblast differentiation by down regulating MyoD activity in a catalytic-independent manner. Cell Signal 2017;35:48-60.

5 Kahn M: Can we safely target the WNT pathway? Nat Rev Drug Discov 2014;13:513-532.

6 Yun SI, Kim HH, Yoon JH, Park WS, Hahn MJ, Kim HC, Chung CH, Kim KK: Ubiquitin specific protease 4 positively regulates the WNT/ $\beta$-catenin signaling in colorectal cancer. Mol Oncol 2015;9:1834-1851.

7 Xing C, Lu XX, Guo PD, Shen T, Zhang S, He XS, Gan WJ, Li XM, Wang JR, Zhao YY, Wu H, Li JM: UbiquitinSpecific Protease 4-Mediated Deubiquitination and Stabilization of PRL-3 Is Required for Potentiating Colorectal Oncogenesis. Cancer Res 2016;76:83-95.

8 Zhang X, Berger FG, Yang J, Lu X: USP4 inhibits p53 through deubiquitinating and stabilizing ARF-BP1. EMBO J 2011;30:2177-2189.

9 Hwang SJ, Lee HW, Kim HR, Lee H, Shin CH, Yun SI, Lee DH, Kim DH, Kim KK, Joo KM, Kim HH: Ubiquitinspecific protease 4 controls metastatic potential through $\beta$-catenin stabilization in brain metastatic lung adenocarcinoma. Sci Rep 2016;6:21596.

10 Xiao N, Li H, Luo J, Wang R, Chen H, Chen J, Wang P: Ubiquitin-specific protease 4 (USP4) targets TRAF2 and TRAF6 for deubiquitination and inhibits TNF $\alpha$-induced cancer cell migration. Biochem J 2012;441:979-986.

11 Fan YH, Yu Y, Mao RF, Tan XJ, Xu GF, Zhang H, Lu XB, Fu SB, Yang J: USP4 targets TAK1 to downregulate TNF $\alpha$-induced NF- $\kappa B$ activation. Cell Death Differ 2011;18:1547-1560.

12 Hou X, Wang L, Zhang L, Pan X, Zhao W: Ubiquitin-specific protease 4 promotes TNF- $\alpha$-induced apoptosis by deubiquitination of RIP1 in head and neck squamous cell carcinoma. FEBS Lett 2013;587:311-316.

13 Kwon YT, Ciechanover A: The Ubiquitin Code in the Ubiquitin-Proteasome System and Autophagy. Trends Biochem Sci 2017;42:873-886.

14 Wei R, Liu X, Yu W, Yang T, Cai W, Liu J, Huang X, Xu GT, Zhao S, Yang J, Liu S: Deubiquitinases in cancer. Oncotarget 2015;6:12872-12889.

15 Liu N, Li X, Huang H, Zhao C, Liao S, Yang C, Liu S, Song W, Lu X, Lan X, Chen X, Yi S, Xu L, Jiang L, Zhao C, Dong X, Zhou P, Li S, Wang S, Shi X, et al.: Clinically used antirheumatic agent auranofin is a proteasomal deubiquitinase inhibitor and inhibits tumor growth. Oncotarget 2014;5:5453-5471.

16 Clerici M, Luna-Vargas MP, Faesen AC, Sixma TK: The DUSP-Ubl domain of USP4 enhances its catalytic efficiency by promoting ubiquitin exchange. Nat Commun 2014;5:5399.

17 Nguyen T, Ho M, Ghosh A, Kim T, Yun SI, Lee SS, Kim KK: An ubiquitin-binding molecule can work as an inhibitor of ubiquitin processing enzymes and ubiquitin receptors. Biochem Biophys Res Commun 2016;479:33-39.

18 Lee BH, Lee MJ, Park S, Oh DC, Elsasser S, Chen PC, Gartner C, Dimova N, Hanna J, Gygi SP, Wilson SM, King RW, Finley D: Enhancement of proteasome activity by a small-molecule inhibitor of USP14. Nature 2010;467:179-184.

19 Rueden CT, Schindelin J, Hiner MC, DeZonia BE, Walter AE, Arena ET, Eliceiri KW: ImageJ2: ImageJ for the next generation of scientific image data. BMC Bioinformatics 2017;18:529.

20 Lin R, Nie J, Ren J, Liang R, Li D, Wang P, Gao C, Zhuo C, Yang C, Li B: USP4 interacts and positively regulates IRF8 function via K48-linked deubiquitination in regulatory T cells. FEBS Lett 2017;591:1677-1686.

21 Li Z, Hao Q, Luo J, Xiong J, Zhang S, Wang T, Bai L, Wang W, Chen M, Wang W, Gu L, Lv K, Chen J: USP4 inhibits p53 and NF- $\kappa B$ through deubiquitinating and stabilizing HDAC2. Oncogene 2016;35:2902-2912.

22 Clevers H, Nusse R: Wnt/ $\beta$-catenin signaling and disease. Cell 2012;149:1192-1205

23 Kahn M: Can we safely target the WNT pathway? Nat Rev Drug Discov 2014;13:513-532.

24 Repetto G, del Peso A, Zurita JL: Neutral red uptake assay for the estimation of cell viability/cytotoxicity. Nat Protoc 2008;3:1125-1131. 
Cellular Physiology Cell Physiol Biochem 2019;53:157-171

\begin{tabular}{rl|l} 
and Biochemistry & $\begin{array}{l}\text { DOI: } 10.33594 / 000000127 \\
\text { Published online: } 29 \text { June } 2019\end{array}$ & $\begin{array}{l}\text { @ } 2019 \text { The Author(s). Published by } \\
\text { Cell Physiol Biochem Press GmbH\&Co. KG }\end{array}$ \\
\cline { 2 - 4 } Nguyen et al.: A Selective Inhibitor of USP4 for the Prevention of Colorectal Cancer
\end{tabular}

25 Chenprakhon P, Sucharitakul J, Panijpan B, Chaiyen P: Measuring binding affinity of protein-ligand interaction using spectrophotometry: binding of neutral red to riboflavin-binding protein. J Chem Educ 2010;87:829-831.

26 Zhu BY, Shang BY, Du Y, Li Y, Li L, Xu XD, Zhen YS: A new HDAC inhibitor cinnamoylphenazine shows antitumor activity in association with intensive macropinocytosis. Oncotarget 2017;8:14748-14758.

27 Reverdy C, Conrath S, Lopez R, Planquette C, Atmanene C, Collura V, Harpon J, Battaglia V, Vivat V, Sippl W, Colland F: Discovery of specific inhibitors of human USP7/HAUSP deubiquitinating enzyme. Chem Biol 2012;19:467-477.

28 Wang X, D’Arcy P, Caulfield TR, Paulus A, Chitta K, Mohanty C, Gullbo J, Chanan-Khan A, Linder S: Synthesis and evaluation of derivatives of the proteasome deubiquitinase inhibitor b-AP15. Chem Biol Drug Des 2015;86:1036-1048. 